\title{
Searches for Higgs bosons and other new physics in scenarios beyond the Standard Model*
}

\author{
David Milstead, on Behalf of the ATLAS and CMS collaborations \\ Fysikum, Stockholm University, Stockholm, Sweden.
}

\begin{abstract}
Searches are presented for Higgs bosons in scenarios beyond the Standard Model by the ATLAS and CMS experiments at the Large Hadron Collider. Searches for other exotic phenomena are also shown.
\end{abstract}

PACS numbers: PACS numbers come here

\section{Introduction}

The Large Hadron Collider (LHC) has opened up the multi-TeV regime for direct searches for particles and physics processes beyond the Standard Model(SM). The ATLAS [1] and CMS [2] collaborations, which have built and maintain multipurpose detectors at the LHC, are at the forefront of this research. To date the major achievement of the LHC experiments has been the discovery of a Higgs-like boson [3, 4], which led to Higgs and Englert being awarded the 2013 Nobel prize for physics ${ }^{1}$. While this is arguably the greatest breakthrough in particle physics for a number of decades there remain a number of open problems which the LHC experiments aim to address. Precision measurements of the new boson are necessary to establish the degree to which the boson's properties are described by mass generation/spontaneous symmetry sector of the SM. Furthermore, there are alternatives to the SM Higgs mechanism which also predict new particles. Additional problems include the apparently unnatural fine-tuning of the Higgs and the absence of a dark matter candidate.

A major goal of the LHC experiments is therefore to explore the $\mathrm{TeV}$-scale for evidence of hitherto unobserved particles and physics processes. Both ATLAS and CMS correspondingly maintain a broad search program. So-called "blue sky" searches look for new phenomena regardless of any predictions of phenomena from models of new physics. A further class of searches follows the predictions of models which extend the SM. Results from the latter case are typically cast in terms of simplified models of new physics.

This paper provides an overview of searches for exotic Higgs searches as well as searches for exotica within non-Higgs scenarios. Owing to the breadth of the search programs and time limitations of the talk which this paper summarises, only a subset of searches is perforce given here. The interested reader is encouraged to see Refs. $[5,6]$ for complete listings of the search outputs of the ATLAS and CMS experiments.

\section{Models of new physics}

A number of theories predict hitherto unseen particles and processes at the $\mathrm{TeV}$ scale. These are described below.

\footnotetext{
* Presented at Matter to the Deepest, Ustron, Poland.

1 The announcement of the Nobel prize winners took place after the talk which these proceedings summarise.
} 


\subsection{Two Higgs Doublet Model}

The Two Higgs Doublet Model [7] (2HDM) extends the Higgs sector of the SM by adding a second doublet. This leads to five physical states: three neutral ( $h, H$ CP-even and $A$ CP-odd) and two charged states $\left(H^{+-}\right)$. Models based on this approach introduce two mixing angles $\alpha$ and $\beta$, which respectively describe the mixing of $\mathrm{CP}$-even and $\mathrm{CP}$ odd states. The quantity $\tan \beta$ is the ratio of the vacuum expectation values of the two doublets. A further classification in these models can be made which depends on the fermion coupling to the Higgs sector. In type I $2 \mathrm{HDM}$ models all quarks couple to just one of the Higgs doublets. In type II models up-type quarks couple to one Higgs doublet and down-type quarks couple to the other. An example of a type II 2HDM is the Minimal Supersymmetric Standard Model [8] (see Section 2.2). Other types (III and IV) consider different coupling for leptons and are not considered here.

\subsection{TeV-scale supersymmetry}

TeV-scale supersymmetry [8] has long been regarded as the most promising of extension of the SM. For an appropriate choice of parameters, it offers an approximate unification of the couplings at the grand unified scale which converge more closely than predicted by the SM [9]. Similarly, while the SM lacks an appropriate dark matter candidate, in R-parity conserving SUSY, the lightest neutralino which could be WIMP dark matter. Finally, SUSY addresses the hierarchy problem albeit leaving a "little hierarchy problem" [10] in its stead. With over 120 free parameters in the most general supersymmetry approach, the Minimal Supersymmetric Standard Model (MSSM), simplified models are therefore used to guide and interpret searches.

The MSSM accommodates a type-II 2HDM. The recently discovered Higgs-like boson with $m \sim 125 \mathrm{GeV}$ can be regarded as the $h$ boson over the bulk of the $m_{A}-\tan \beta$ region which is not excluded by direct MSSM Higgs boson searches. It can also be regarded as the $\mathrm{H}$ particle if the charged Higgs boson mass is below $150 \mathrm{GeV}$ and $\tan \beta$ is small. Because of enhanced couplings of the MSSM Higgs bosons to $b$ and $t$ quarks and tau leptons for large $\tan \beta$ most searches for these bosons exploit associated Higgs production with $b$ and $t$ quarks and decay modes involving $b, t$ and $\tau$ particles.

\subsection{Searches for new particles in the Two-Higgs-Doublet-Model}

The ATLAS experiment has performed a search for Higgs bosons in the Two-HiggsDoublet approach using the decay $H \rightarrow W W \rightarrow e \nu \mu \nu$ [11]. The analysis is based on data taken at $8 \mathrm{TeV}$ centre-of-mass energy and corresponds to an integrated luminosity of $13 \mathrm{fb}^{-1}$. A neural network technique was exploited to increase the search sensitivity. Figure 1 shows the discriminant distribution for data and background expectations for a second scalar of mass $240 \mathrm{GeV}$. The search found no evidence for a second scalar boson in the mass range between 135 and $300 \mathrm{GeV}$. This allowed limits to be set of type-I and typeII Two-Higgs-Doublet-Models which depend on the scalar mass and the mixing angles $\alpha$, $\beta$.

\subsection{Searches for Higgs particles in the MSSM}

The ATLAS and CMS experiments have made a number of searches for anomalous Higgs production which are inspired by or can be interpreted within the MSSM $[12,13]$. The ATLAS experiment made a search for neutral Higgs bosons within the MSSM using data taken at $7 \mathrm{TeV}$ centre-of-mass energy for an integrated luminosity of $4.7 \mathrm{fb}^{-1}$ [12]. Decays into oppositely signed muon or tau lepton pairs were sought, also using $b$-tagging 


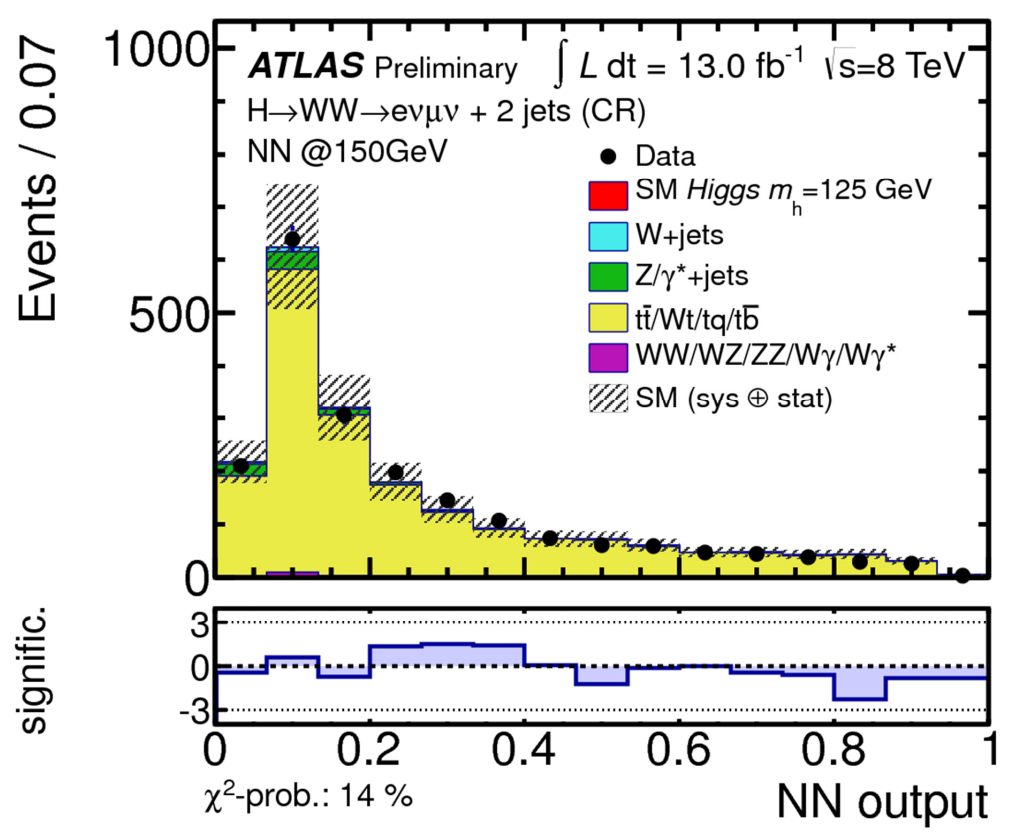

Fig. 1. Discriminant distributions for a neutral network-based search which is optimised for $m_{H}=$ $240 \mathrm{GeV}$ in the 2-jet channel. Data and background expectations are shown.

information. CMS made a similar search [13], focusing on the decay to tau leptons and using data taken at $7 \mathrm{TeV}$ and $8 \mathrm{TeV}$ centre-of-mass energies (Figure 2).

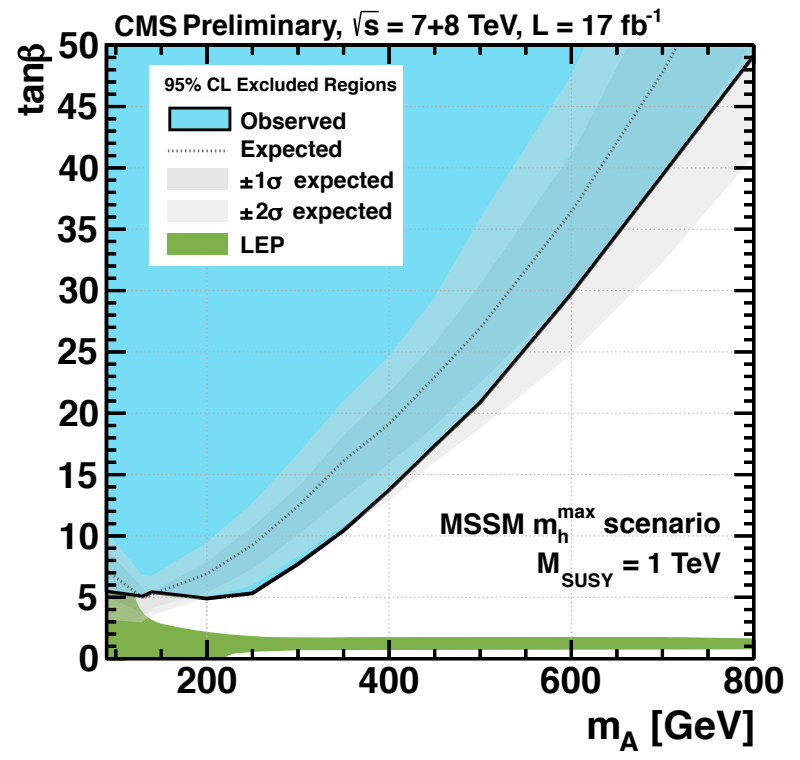

Fig. 2. Exclusion limits in $\tan \beta$ and $m_{A}$ space from searches for neutral Higgs decaying to tau pairs within the MSSM framework. 
The CMS experiment has performed a dedicated search for Higgs decaying via $H \rightarrow$ $b \bar{b}$ [14]. Multijet final states were considered with three jets identified as coming from $b$-quarks. At least one jet may contain a non-isolated muon. Signals are sought in states characterised either purely by jets ("hadronic") or with an additional non-isolated muon ("semi-leptonic"). The sought signature, for both types of states, is a peak in the invariant mass distribution of the two leading $b$-jets over the large multijet background. Different methods are used to estimate the background for each state. No signal was observed and limits were extracted. Constraints on the $\tan \beta$ vs $M_{A}$ plane are shown in Figure 3.

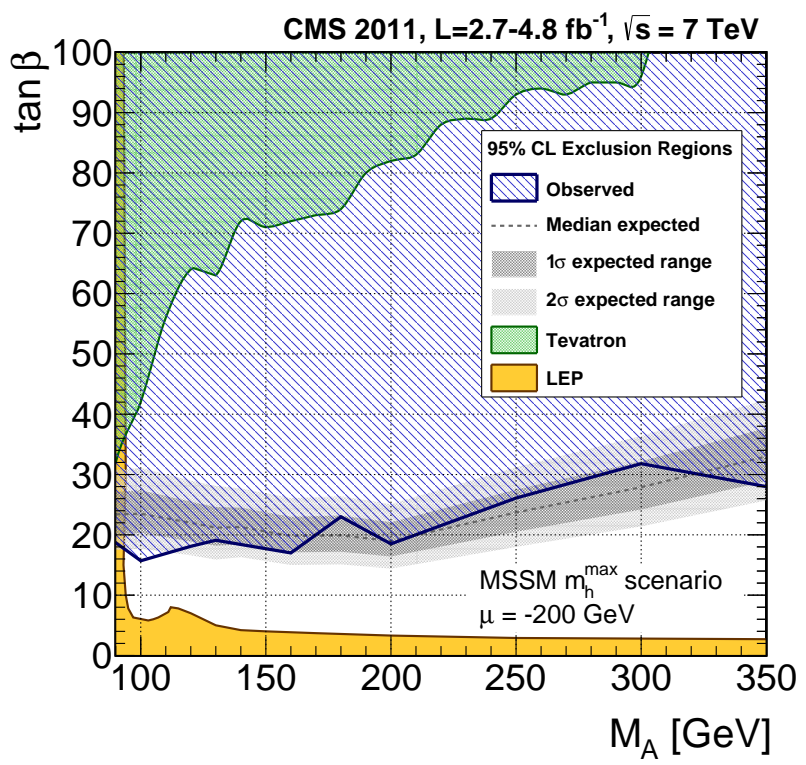

Fig. 3. Exclusion limits in $\tan \beta$ and $m_{A}$ space from searches for $H \rightarrow b \bar{b}$.

\subsection{Unobservable Higgs Decays}

In a number of scenarios the Higgs can decay to objects which pass through the detector without interacting. A promising means of searching for so-called invisible Higgs decays is to look for associated Higgs production with a $Z$-boson and the process $Z H \rightarrow l l+$ invisible. In this scenario, the main signature is a pair of oppositely sign leptons with a mass near the $Z$-pole and missing transverse energy. No significant excesses were observed in the ATLAS and CMS analyses $[15,16]$ and limits on the cross section times branching

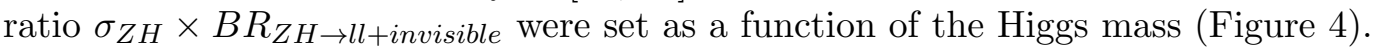

\section{Non-Higgs searches for physics beyond the Standard Model}

It is impossible to do justice to the breadth of the exotics physics programs of the ATLAS and CMS experiments in these proceedings. Instead, representative results have been selected. However, Figure 5 shows the range of searches which have been made (by the CMS experiment) for a number of non-SUSY scenarios. Limits within various scenarios are also shown. As can be seen, even with the comparatively modest amounts of luminosity collected by the experiments (in relation to the full luminosity to be delivered during the LHC lifetime) mass limits in excess of $1 \mathrm{TeV}$ are already being made in certain scenarios. Similar results from ATLAS can be found in Ref. [17]. 

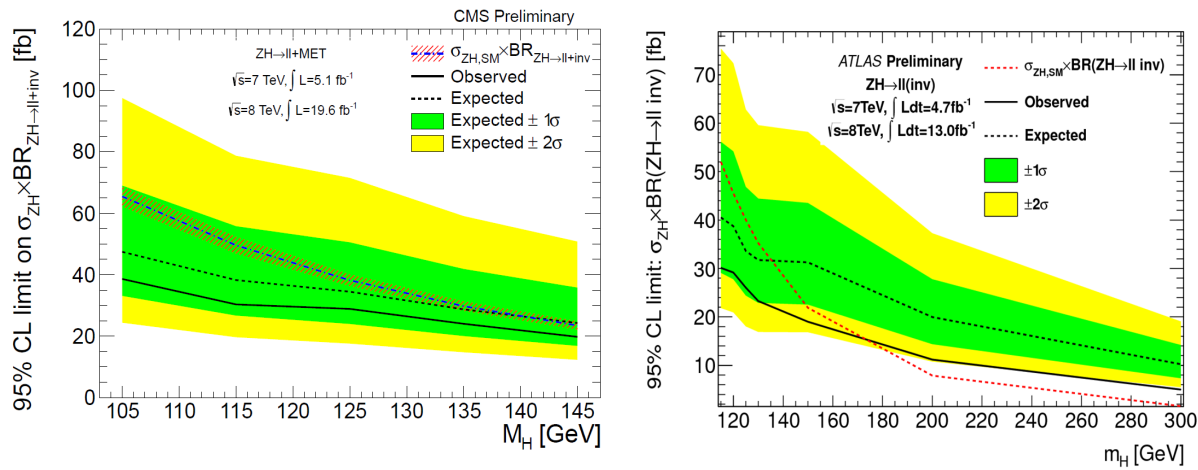

Fig. 4. Exclusion limits for invisible Higgs decays.

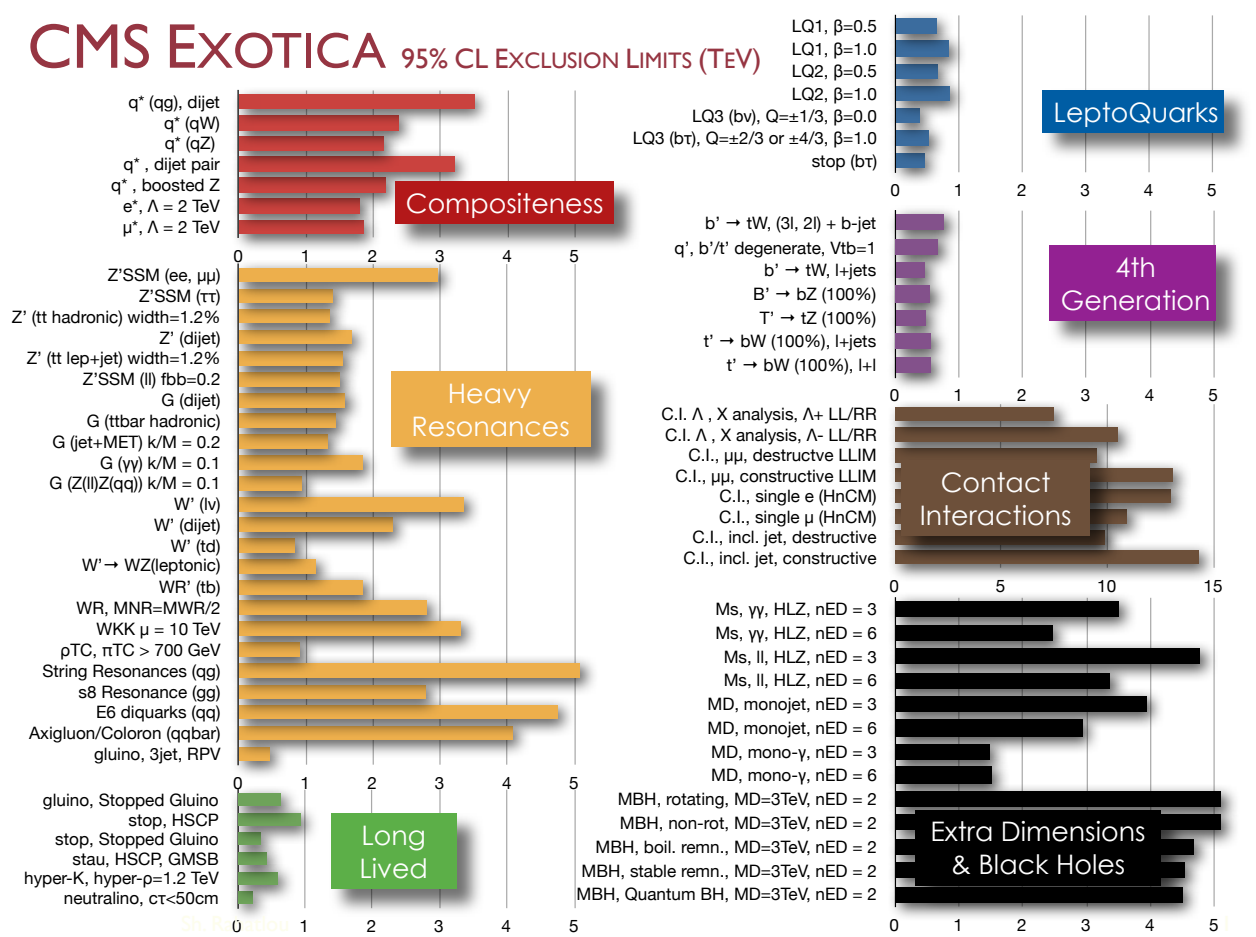

Fig. 5. Compilation of results on searches for physics beyond the SM, interpreted within nonsupersymmetry models.

\subsection{Searches for dark matter}

The LHC is potentially a dark matter factory. Processes such as monojet and monophoton production could be accompanied by the emission of a WIMP-like object (and thus 
missing transverse energy). The ATLAS and CMS experiments have made a number of searches for this phenomena (eg. [18, 19, 21, 20, 22]). A representative search is the measurement of the rate of processes in which which a $W$ or $Z$ is produced and subsequently decays hadronically [22]. Selections are made for transverse energy and a jet with a mass consistent with a $W$ or $Z$ boson. No events are observed beyond those expected from background estimations. This allows limits to be set both on the production cross section and, with additional phenomenological input, the WIMP-nucleon cross section as a function of WIMP mass. This limit is shown in Figure 6 (for spin-independent interactions). The ATLAS results can be compared directly to those from direct WIMP detection experiments. The LHC currently provides good sensitivity for masses below $\sim 10 \mathrm{GeV}$.

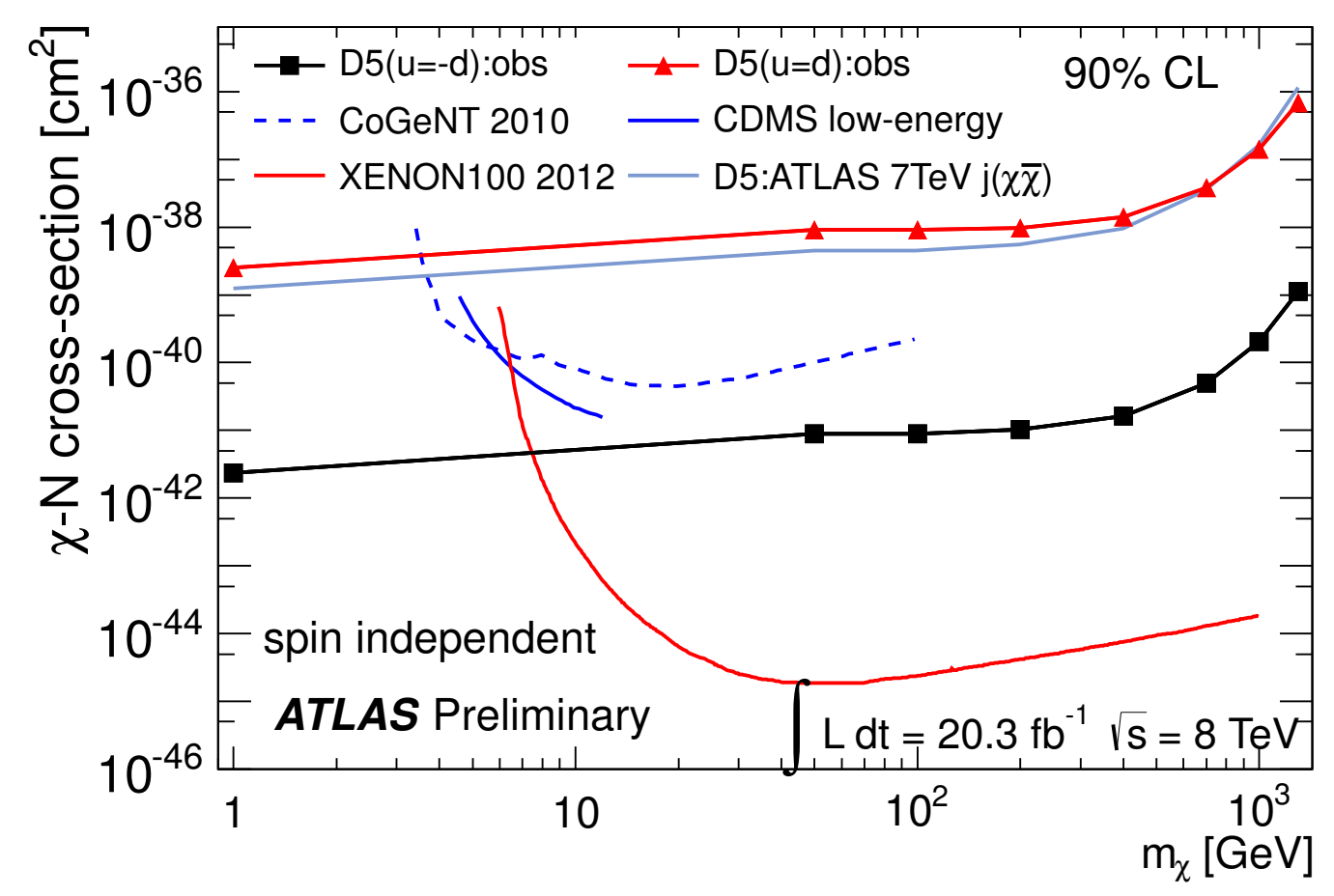

Fig. 6. Limits on the WIMP nucleon cross section as a function of WIMP mass.

\subsection{Searches for new resonances}

Resonances decaying into a range of final states have been sought. A representative search is that made by ATLAS for dilepton resonances [23]. Following selections for good quality reconstructed lepton candidates exceeding minimum energy thresholds, oppositely signed dielectron and dimuon mass spectra are studied. The dielectron spectra is shown in Figure 7, along with predictions for exotic heavy bosons (Z') of various masses decaying to dielectrons.

\subsubsection{Magnetically charged objects}

Magnetic monopoles are routinely sought at new colliders [24]. ATLAS and CMS are sensitive to a wide range of magnetic charges [25] including stopped searches [26]. Signatures of a magnetic monopole with the Dirac charge include high ionisation and narrow clusters in an electromagnetic calorimeter. The ATLAS experiment has exploited this to look for monopoles. Figure 8 shows the distribution of the fraction of high threshold hits 


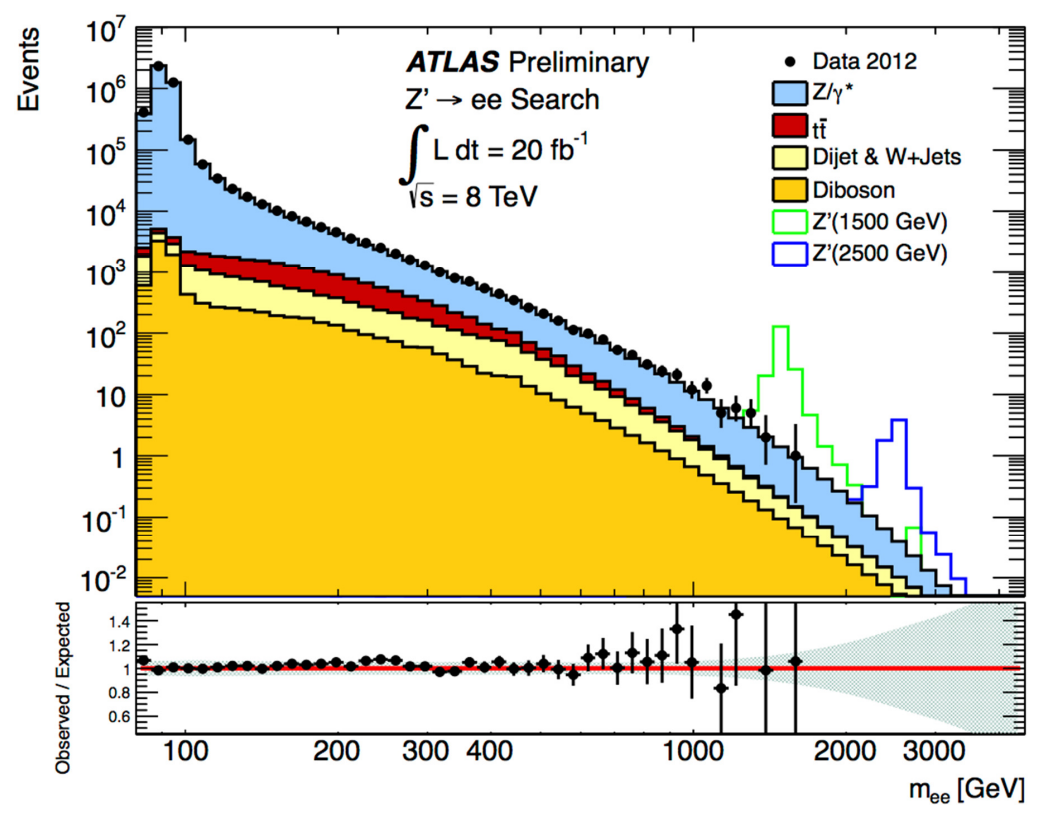

Fig. 7. Dielectron invariant mass compared with backgrounds and two examples of exotic resonances (Z' bosons). The ratio of observed to expected events is also shown.

in the Transition Radition Tracker $\left(f_{H T}\right)$, which increases with the amount of deposited ionisation energy, versus an electromagnetic shower shape variable $\left(\sigma_{R}\right)$, which decreases as the cluster gets narrower. Collision data are shown, as are Monte Carlo simulated data from a model of Dirac monopole production. There is a clear separation between the two data samples and no evidence for Dirac monopoles. This leads to an upper limit on the production of monopoles of $2 \mathrm{pb}$.

\subsubsection{Long-lived penetrating particles}

Heavy stable leptons, such as stable staus in SUSY, would propagate through the detector like a heavy muon. Massive coloured particles would hadronise; in the context of SUSY the heavy hadrons are referred to as $R$-hadrons. Interactions of $R$-hadron in matter involve electric charge exchange which implies that $R$-hadrons can appear as heavy penetrating muons or may be unchanged (or flip charge) in different parts of the detector $[27,28]$. Searches take this into account by looking for signals in various detector regions. The CMS collaboration has published stringent limits on stable sleptons as well as $R$-hadrons acting like muon and non-muon like objects (Figure 9 ). To a 95\% C.L. stable staus with masses up to around $300 \mathrm{GeV}$, and $R$-hadrons with masses up to $1.3 \mathrm{TeV}$ are excluded. The $R$-hadron limits depend on the type of heavy coloured object and assumptions regarding how they interact. Limits on direct production of objects with charge $\frac{2 e}{3}$ are also shown. For a Drell-Yan production model, masses of less than around $200 \mathrm{GeV}$ are ruled out. 


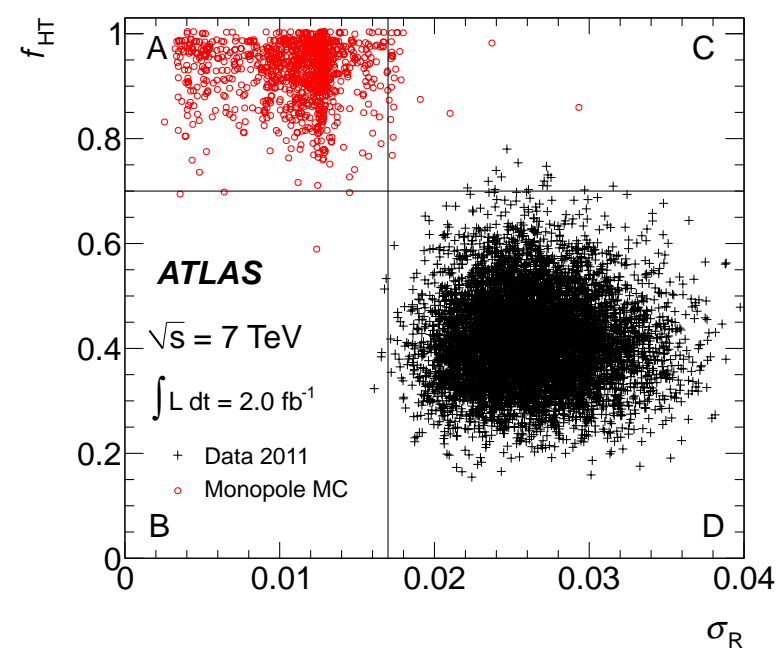

Fig. 8. Distribution of $f_{H T}$ versus $\sigma_{R}$. Data (black, closed circles) and the prediction from Dirac monopoles (red, open circles) are shown.

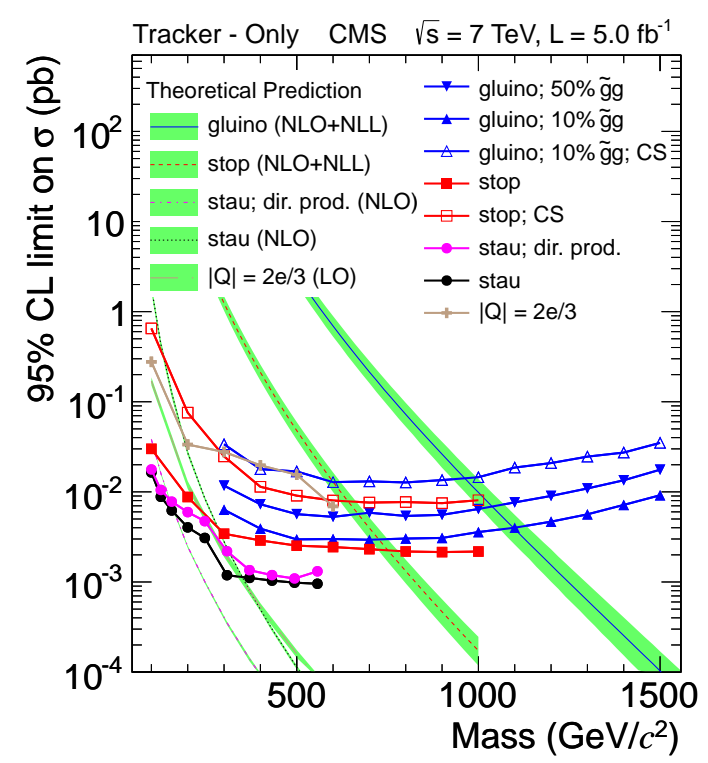

Fig. 9. Upper limits on the production cross sections on various putative types of massive long-lived particles.

\subsection{Searches for supersymmetry}

Supersymmetry is arguably the leading extension to the SM in the literature. It stablises the Higgs mass and offers a solution to the naturalness problem as well providing an intriguing approximate coupling unification around the grand unified scale. However, there are no unambiguous signals of SUSY so a wide range of searches for possible signatures have been made. The body of R-parity conserving searches from ATLAS with a WIMP-candidate as the lightest supersymmetric particle are summarised in Figure 10. As can be seen the strong production signatures show the strongest limits and the weak production scenarios offer comparatively modest limits at present. Similar results from 
CMS can be found in Ref. [29].

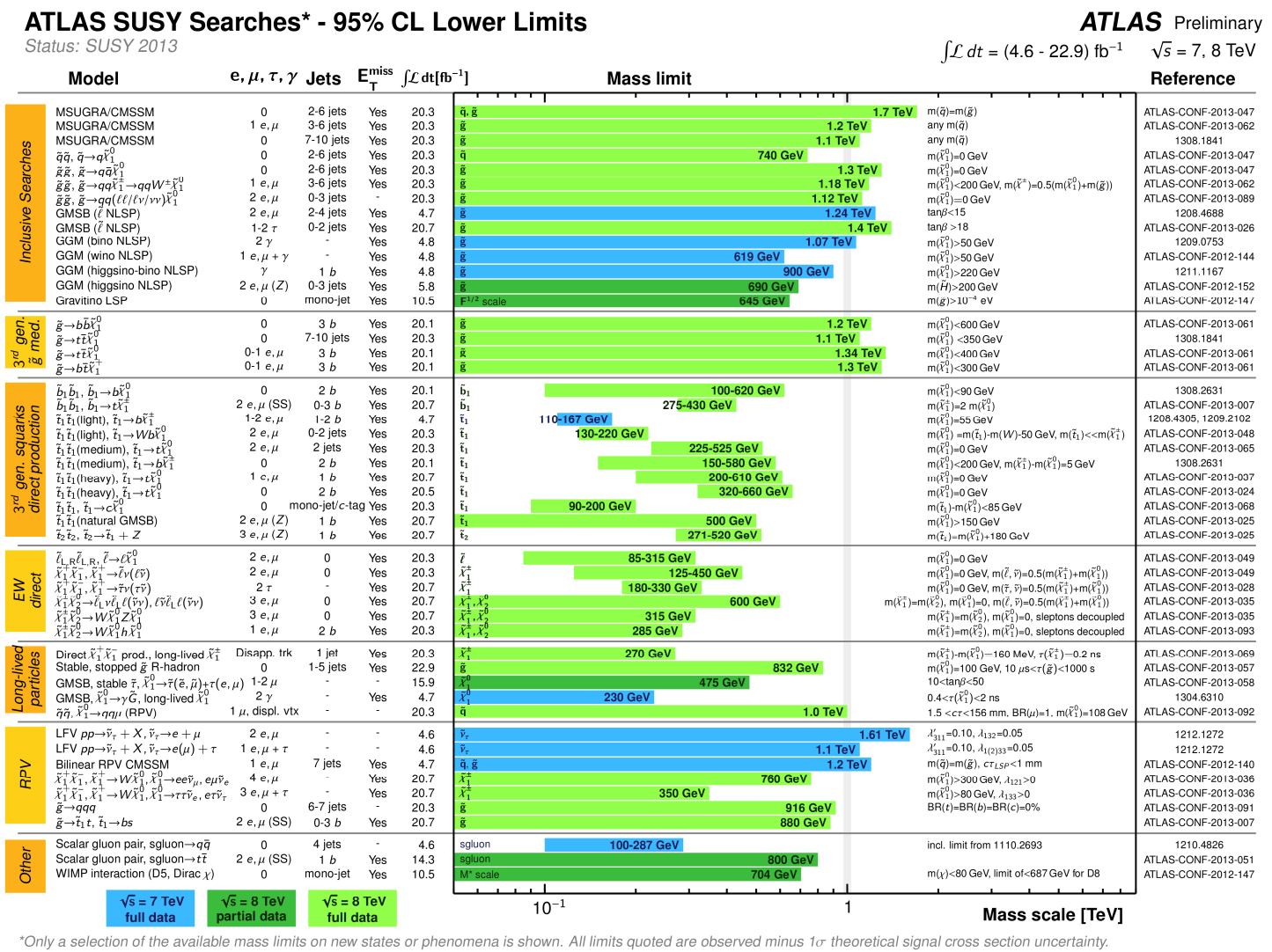

Fig. 10. Compilation of ATLAS results on searches for signatures of supersymmetry.

\section{Summary}

A selection of results from ATLAS and CMS on searches for physics beyond the Standard Model in the Higgs and non-Higgs sectors has been shown.

\section{REFERENCES}

[1] ATLAS Collaboration, JINST 3 (2008) S08003.

[2] CMS Collaboration, S. Chatrchyan et al., JINST 3 (2008) S08004.

[3] ATLAS Collaboration, Phys. Lett. B 716 (2012) 1 [arXiv:1207.7214 [hep-ex]].

[4] CMS Collaboration, S. Chatrchyan et al., JHEP 1306 (2013) 081 [arXiv:1303.4571 [hep-ex]].

[5] https://twiki.cern.ch/twiki/bin/view/AtlasPublic

[6] https://twiki.cern.ch/twiki/bin/view/CMSPublic/PhysicsResults

[7] T. D. Lee, Phys. Rev. D 8 (1973) 1226.

[8] H. P. Nilles, Phys. Rept. 110 (1984) 1. 
[9] N. Arkani-Hamed, A. Gupta, D. E. Kaplan, N. Weiner and T. Zorawski, arXiv:1212.6971 [hep$\mathrm{ph}$.

[10] H. -C. Cheng and I. Low, JHEP 0309 (2003) 051 [hep-ph/0308199].

[11] ATLAS Collaboration, ATLAS-CONF-2013-027 (2013), http://cds.cern.ch/record/ 1525887.

[12] ATLAS Collaboration, arXiv:1211.6956 [hep-ex].

[13] CMS Collaboration, CMS-PAS-HIG-12-050 (2012), http://cds .cern.ch/record/1493521.

[14] CMS Collaboration, S. Chatrchyan et al., Phys. Lett. B 722 (2013) 207 [arXiv:1302.2892 [hep-ex]].

[15] CMS Collabration, CMS-PAS-HIG-13-018 (2013), http://cds.cern.ch/record/1561758.

[16] ATLAS Collaboration, ATLAS-CONF-2013-011 (2013), http://cds.cern.ch/record/ 1523696.

[17] https://twiki.cern.ch/twiki/bin/view/AtlasPublic/CombinedSummaryPlots\# ExoticsSummary.

[18] CMS Collaboration, CMS-PAS-EXO-12-048 (2012), http://cds.cern.ch/record/1525585.

[19] CMS Collaboration, S. Chatrchyan et al., Phys. Rev. Lett. 108 (2012) 261803 [arXiv:1204.0821 [hep-ex]].

[20] ATLAS Collaboration, ATLAS-CONF-2012-147 (2012) http://cds.cern.ch/record/ 1493486.

[21] ATLAS Collaboration, Phys. Rev. Lett. 110 (2013) 011802 [arXiv:1209.4625 [hep-ex]].

[22] ATLAS Collaboration, ATLAS-CONF-2013-073 (2013) http://cds.cern.ch/record/ 1562926.

[23] ATLAS Collaboration, ATLAS-CONF-2013-017 (2013) http://cds.cern.ch/record/ 1525524.

[24] M. Fairbairn et al., Phys. Rept. 438 (2007) 1 [hep-ph/0611040].

[25] A. De Roeck et al., Eur. Phys. J. C 72 (2012) 1985 [arXiv:1112.2999 [hep-ph]].

[26] A. De Roeck et al., Eur. Phys. J. C 72 (2012) 2212.

[27] Y. R. de Boer et al., J. Phys. G 35 (2008) 075009 [arXiv:0710.3930 [hep-ph]].

[28] R. Mackeprang and D. Milstead, Eur. Phys. J. C 66 (2010) 493 [arXiv:0908.1868 [hep-ph]].

[29] https://twiki.cern.ch/twiki/bin/view/CMSPublic/PhysicsResultsSUS. 